\title{
The wide-aperture gamma-ray telescope TAIGA-HiSCORE in the Tunka Valley: Design, composition and commissioning
}

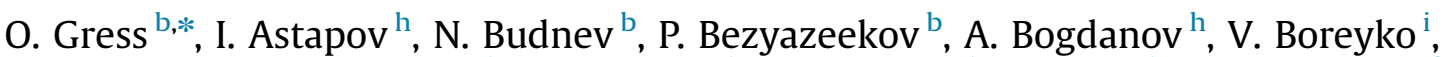 \\ M. Brückner ${ }^{\mathrm{j}}$, A. Chiavassa ${ }^{\mathrm{d}}, \mathrm{O}$ Chvalaev $^{\mathrm{b}}$, A. Dyachok $^{\mathrm{b}}$, T. Gress ${ }^{\mathrm{b}}$, S. Epimakhov ${ }^{\mathrm{f}}$, \\ E. Fedoseev ${ }^{b}$, A. Gafarov ${ }^{b}$, N. Gorbunov ${ }^{i}$, V. Grebenyuk ${ }^{i}$, A. Grinuk ${ }^{i}$, O. Grishin ${ }^{b}$, D. Horns ${ }^{f}$,
} A. Ivanova ${ }^{\text {b }, ~ A . ~ K a l i n i n ~}{ }^{i}$, N. Karpov ${ }^{\text {a }}$, N. Kalmykov ${ }^{\text {a }}$, Yu. Kazarina ${ }^{\text {b }, ~ N . ~ K i r i c h k o v ~}{ }^{\text {, }}$, S. Kiryuhin ${ }^{b}$, R. Kokoulin ${ }^{\text {h }}$, K. Komponiest ${ }^{\text {h }}$, E. Korosteleva ${ }^{a}$, V. Kozhin ${ }^{a}$, M. Kunnas ${ }^{f}$, L. Kuzmichev ${ }^{\text {a }}$, V. Lenok ${ }^{\text {b }}$, B. Lubsandorzhiev ${ }^{c}$, N. Lubsandorzhiev ${ }^{\text {a }}$, R. Mirgazov ${ }^{\text {, }}$, R. Mirzoyan ${ }^{\text {bee }}$, R. Monkhoev ${ }^{\text {b }}$, R. Nachtigall ${ }^{f}$, A. Pakhorukov ${ }^{b}$, M. Panasyuk ${ }^{a}$, L. Pankov ${ }^{b}$, A. Petrukhin $^{\text {h }}$, V. Platonov ${ }^{\text {b }}$, V. Poleschuk ${ }^{\mathrm{b}}$, E. Popova ${ }^{\mathrm{a}}$, A. Porelli $^{\mathrm{g}}$, V. Prosin ${ }^{\mathrm{a}}$, , G. Rubtsov $^{\mathrm{c}}$, A. Pushnin $^{\text {b }}$, V. Samoliga ${ }^{\text {b }}$, A. Saunkin ${ }^{\text {b }}$, Yu. Semeney ${ }^{\text {b }}$, B. Shaibonov $(j u)^{\text {c }}$, A. Silaev ${ }^{\text {a }}$, A. Silaev(ju) ${ }^{a}$, A. Skurikhin ${ }^{\text {a }}$, V. Slucka ${ }^{i}$, C. Spiering ${ }^{g}$, L. Sveshnikova ${ }^{\text {, }}$, V. Tabolenko ${ }^{\text {b }}$, B. Tarashchansky ${ }^{\mathrm{b}}$, A. Tkachenko ${ }^{\mathrm{i}}$, L. Tkachev ${ }^{\mathrm{i}}$, M. Tluczykont ${ }^{\mathrm{f}}$, D. Voronin $^{\mathrm{b}}$, R. Wischnewski ${ }^{\text {g }}$, A. Zagorodnikov ${ }^{\text {b }}$, V. Zurbanov ${ }^{\text {b }}$, I. Yashin ${ }^{\text {h }}$

\footnotetext{
a Skobeltsyn Institute of Nuclear Physics MSU, Moscow, Russia

${ }^{\mathrm{b}}$ Institute of Applied Physics ISU, Irkutsk, Russia

c Institute for Nuclear Research of RAN, Moscow, Russia

${ }^{\mathrm{d}}$ Dipartimento di Fisica Generale Universiteta di Torino and INFN, Torino, Italy

e Max-Planck-Institute for Physics, Munich, Germany

${ }^{\mathrm{f}}$ Institut fr Experimentalphysik, University of Hamburg, Germany

g DESY, Zeuthen, Germany

${ }^{\mathrm{h}}$ NRNU MEPhI, Moscow, Russia

i JINR, Dubna, Russia

${ }^{\mathrm{j}}$ Institute for Computer Science, Humboldt-University, Berlin, Germany
}

\section{A R T I C L E I N F O}

\section{Article history:}

Received 20 July 2016

Accepted 11 August 2016

Available online 12 August 2016

Keywords:

Cosmic ray

Gamma-ray sources

Cherenkov light

Data acquisition system

\begin{abstract}
A B S T R A C T
The new TAIGA-HiSCORE non-imaging Cherenkov array aims to detect air showers induced by gamma rays above $30 \mathrm{TeV}$ and to study cosmic rays above $100 \mathrm{TeV}$. TAIGA-HiSCORE is made of integrating air Cherenkov detector stations with a wide field of view $(0.6 \mathrm{sr})$, placed at a distance of about $100 \mathrm{~m}$. They cover an area of initially $\sim 0.25 \mathrm{~km}^{2}$ (prototype array), and of $\sim 5 \mathrm{~km}^{2}$ at the final phase of the experiment. Each station includes 4 PMTs with 20 or $25 \mathrm{~cm}$ diameter, equipped with light guides shaped as Winstone cones. We describe the design, specifications of the read-out, DAQ and control and monitoring systems of the array. The present 28 detector stations of the TAIGA-HiSCORE engineering setup are in operation since September 2015.
\end{abstract}

(c) 2016 Elsevier B.V. All rights reserved.

\section{Introduction}

The $\gamma$-ray detection beyond $10 \mathrm{TeV}$ is extremely important for the search for the most energetic Galactic accelerators. The energy spectra of most known $\gamma$-ray sources only reach up to few tens of

\footnotetext{
* Corresponding author.

E-mail address: grol08@rambler.ru (O. Gress).
}

$\mathrm{TeV}$ and to $80 \mathrm{TeV}$ from the Crab Nebula. Uncovering spectral shape of the $\gamma$-ray sources up to few $100 \mathrm{TeV}$ could answer the question whether some of these objects are cosmic ray Pevatrons, i.e. Galactic PeV accelerators [1]. Due to the typical powerlaw shape of the energy spectra of cosmic $\gamma$-ray sources, large effective detection areas are needed in order to access higher energies.

The TAIGA-HiSCORE array is part of the gamma-ray observatory TAIGA (Tunka Advanced Instrument for cosmic ray physics and 
Gamma Astronomy). The TAIGA is currently under construction in the Tunka valley, about $50 \mathrm{~km}$ from Lake Baikal in Siberia, Russia [2]. The key advantage of the TAIGA will be the hybrid detection of EAS Cherenkov radiation by the wide-angle detector stations of the TAIGA-HiSCORE array and by the Imaging Air Cherenkov Telescopes of the TAIGA-IACT array [3]. TAIGA comprises also the Tunka-133 array and will furthermore host up a net of surface and underground stations for measuring the muon component of air showers.

The principle of the TAIGA-HiSCORE detector follows the idea [4]: the detector stations measure the light amplitudes and full time development of the air shower ligth front up to distances of several hundred meters from the shower core.

\section{Design of the gamma-ray telescope TAIGA-HiSCORE}

Currently TAIGA-HiSCORE array is composed of 28 detector stations distributed in a regular grid over a surface area of $0.25 \mathrm{~km}^{2}$ with an inter-station spacing of about $106 \mathrm{~m}$ (see Fig. 1).

The optical stations was deployed in 2014 on the same site in the Tunka Valley $\left(\varphi=51^{\circ} 48^{\prime} 47.5^{\prime \prime} \mathrm{N}, \lambda=103^{\circ} 04^{\prime} 16.3^{\prime \prime} \mathrm{E}, h=675 \mathrm{~m}\right.$ a.s.l.) where the Tunka-133 installation is located [5]. All stations are tilt into the southern direction by up to $25^{\circ}$ to increase the accessible night sky area for study $\gamma$-quanta fluxes from the first test objective - Crab nebula.

\section{Optical station}

Optical Station is a metal box (size of $1 \times 1 \times 1 \mathrm{~m}^{3}$ ) with remotely-operated lid to protect against sunlight, atmospheric precipitations and dust (see Fig. 2).

This metal box contains the optical station controller, high voltage power supply unit (HV), heaters for the plexiglass input windows, photomultipliers with voltage dividers and preamplifiers. Each optical station contains four large area photomultipliers with 20 or $25 \mathrm{~cm}$ diameter, namely EMI ET9352KB, or Hamamatsu R5912 and R7081. Each PMT has a Winston cone (made of ten segments of ALANOD 4300UP foil with reflectivity $80 \%$ ) with $0.4 \mathrm{~m}$ diameter and $30^{\circ}$ viewing angle (field of view of $\sim 0.6 \mathrm{sr}$ ). Plexiglass is used on top to protect the PMTs against dust and humidity. So a total station light collection area is $0.5 \mathrm{~m}^{2}$. We use the six-stage divider of PMT that has a nominal gain of $10^{4}$ at $1.4 \mathrm{kV}$ supply. The fast pulse preamplifiers for anode and dynode sygnals are designed on base of the ultrahigh speed current feedback amplifier AD8009 chip.

Average PMT anode current due to night sky background light (NSB) is $\sim 80 \pm 30 \mu \mathrm{A}$ depending on the detector operating mode and the weather conditions. Shower counting rate is $\sim 10 \mathrm{~Hz}$ by station trigger threshold $\sim 200$ photoelectrons. Since the average quantum efficiency is $\sim 0.1$ for the optical station, the Cherenkov light detection threshold per station is $\sim 0.3$ photons $/ \mathrm{cm}^{2}$.

\section{Electronics of the array}

The electronics of the array for functional purpose consists of three parts: data acquisition (DAQ) system, slow control system and monitoring system. The basic electronics components, their interrelationship and location shown in Fig. 3.

The Heating Controller maintains a stable temperature DAQ into the Electronics Box.

\subsection{Slow control system}

The main elements of the slow control system Fig. 4 are the three controllers each on the basis 16-Bit Flash Microcontroller PIC24FJ64GA004I-PT. This controllers (HV Controller, Measurement Controller and Power Load Controller) are connected in single board. This controllers board is located directly in the optical station and connected to the MOXA NPort5150A converter via RS-485 bus. An 8-Port Gigabit L2 Managed Switch TL-SG3210 with 2 SFP Slots and the Moxa NPort5150A Serial Ethernet Converter are placed in the Electronics Box, with special temperature control (see Fig. 3).

The slow control system is designed to:

- control lid position of the station;

- control heating of the input plexiglass windows;

- monitoring load currents of the lid motor and heating;

- control HV power supply of PMTs;

- monitoring HV and PMT anode currents;

- auto turn-off by overcurrent;

- auto turn-off by time of the end of the night.
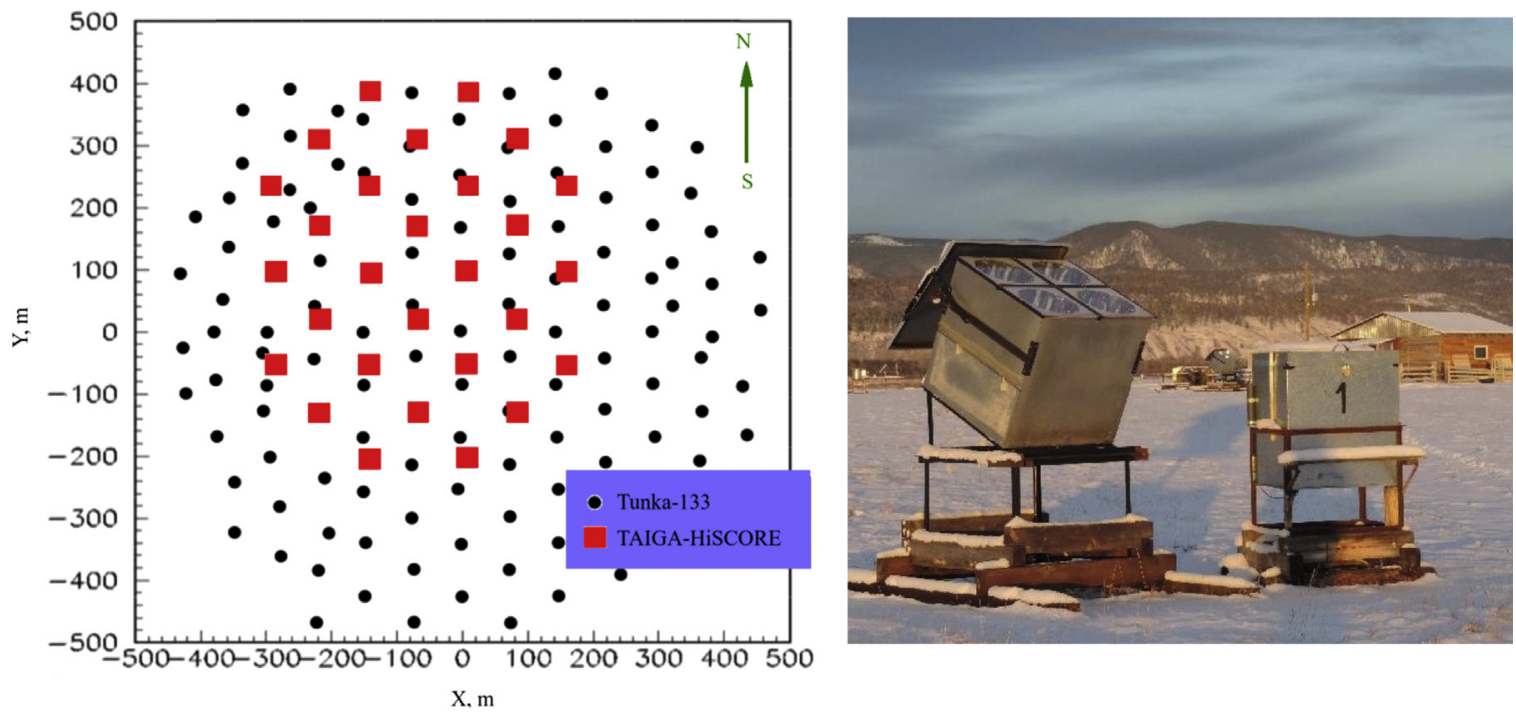

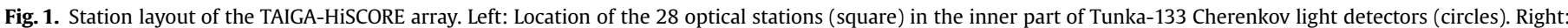
Photo of the optical station and its electronic box. In the background - the central DAQ building. 


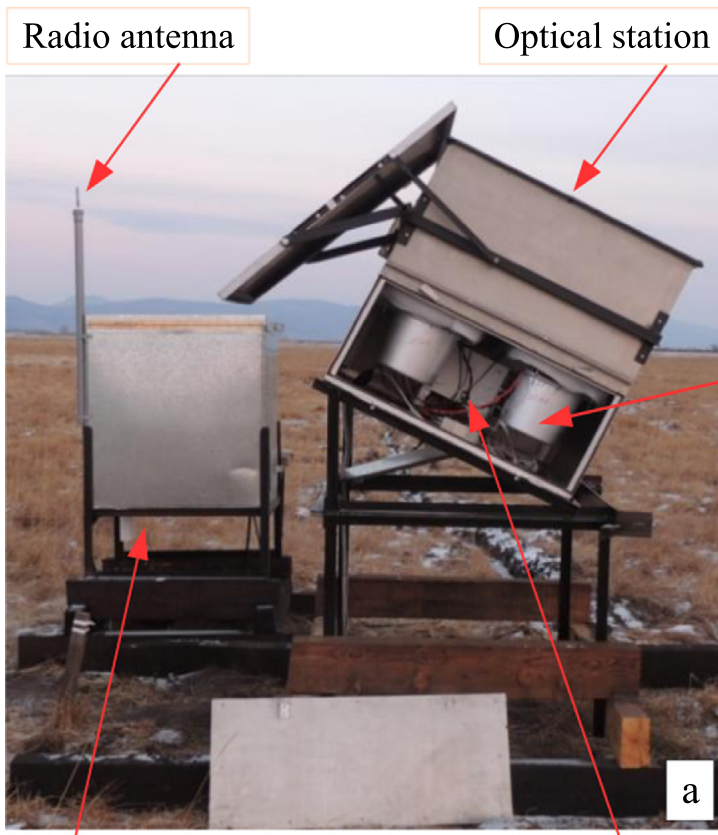

Electronics box
Controller

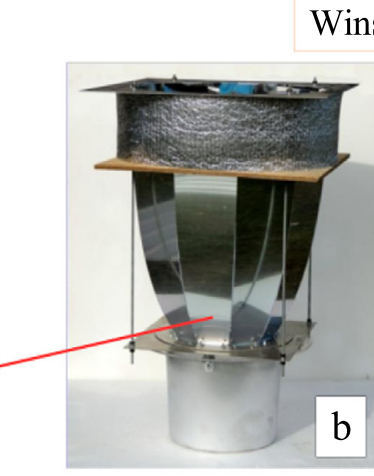

Winston cone

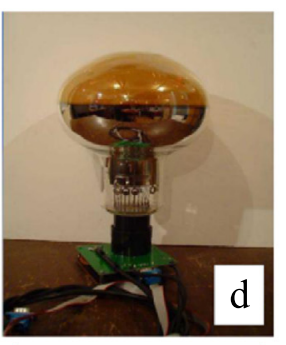

PMT R5912
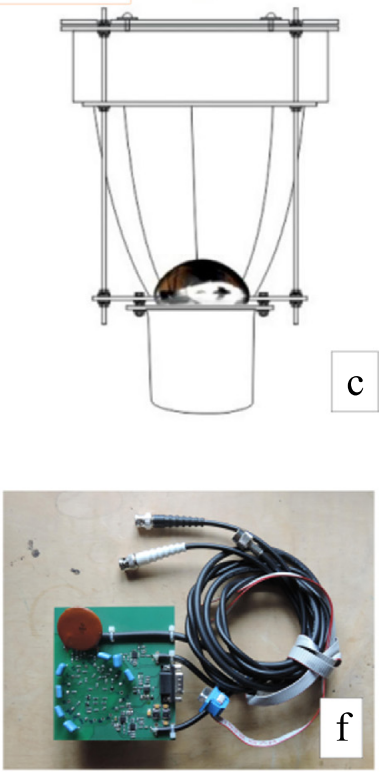

HV divider

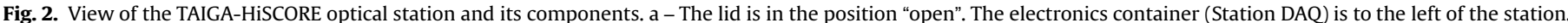

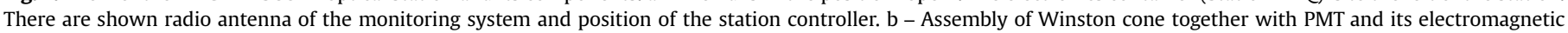

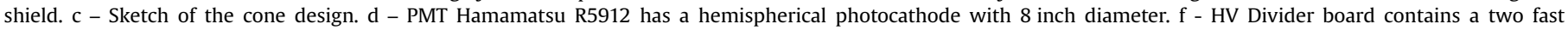
preamplifier (for anode and dynode signals).

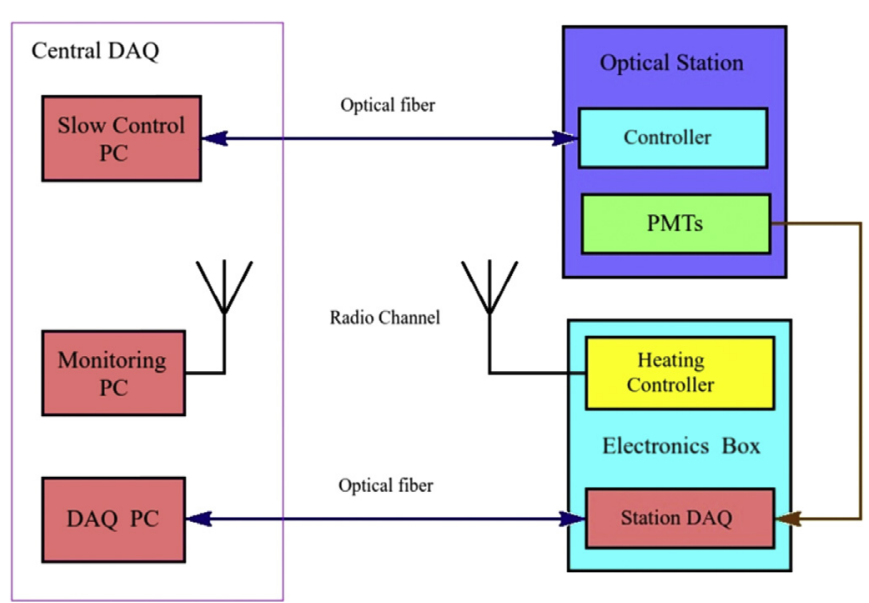

Fig. 3. Block diagram of the basic electronics units of the TAIGA-HiSCORE array. PMTs and Controller are located in the optical station. The Controller consists of three units (HV, Measurement and Power Load controllers) and it monitors and controls the Optical Station. Heating Controller provides thermostabilization and allows to monitor both temperature and also power supply of the Station DAQ using radio communication.

The slow control system provides data communication between optical station controllers and main control program of the personal computer. The software for the slow control system was written in the development environment LabVIEW.

\subsection{Monitoring system}

The main functions of the monitoring system are remote monitoring of the temperature and current consumption of the electronics in electronics boxes during its operation. Auxiliary functions are the temperature changing at which the controller turns on power to the station DAQ setting temperature of the switch on/off heater and fan of cooling, possibility switch on/off power supply of the station DAQ by the operator.

The monitoring system includes a Heating Controller Fig. 3 with connected XBee-PRO ZB RF Module router located at each station, and a computer in the central DAQ to the connected XBeePRO ZB RF Module coordinator via USB-connector. Heating Controller is designed on the basis 16-Bit Flash Microcontroller PIC24FJ64GA004I-PT to control the power load (on/off power, heating and cooling). The XBee-PRO ZB RF Modules [6] operate within the ISM $2.4 \mathrm{GHz}$ frequency band with a low data rate (250 Kbps).

\subsection{Data acquisition system}

The DAQ system is constructed of two parts: Central DAQ located in the main building of the data collection and storage and Station DAQ in the electronics box near the optical detector (see Fig. 5). These two DAQ parts are interconnected by single-mode fiber optic cables, with a length of $100-700 \mathrm{~m}$ depending on the distance from the main building.

Stations detect air showers independently. The station trigger is built using the sum of the 4 analog anode signals in each station. Using the analog signals summation from the four PMTs allows a twofold reduction of the air shower registration threshold, compared to one PMT.

The main 8 channels DRS- 4 board of the Station DAQ is designed on the basis of DRS-4 (Domino Ring Sampler) chip [7] and FPGA Xilinx Spartan-6. The anode and dynode pulses outputs are used for improved dynamic range. The time step of PMT signals digitization is $0.5 \mathrm{~ns}$ (sampling frequency $2 \mathrm{GHz}$ ) and read-out window is $0.5 \mu$ s (1024 cells). The DRS- 4 board has a 14-bit amplitude resolution and an input signal range $\pm 2 \mathrm{~V}$ by each channel. Data are read out using an Ethernet interface to the DRS-4 board and sent via optical fiber link to a Central DAQ PC.

The Host-DRS is synchronization unit for all optical stations. It provides an event synchronization precision of $0.2 \mathrm{~ns}$ and 


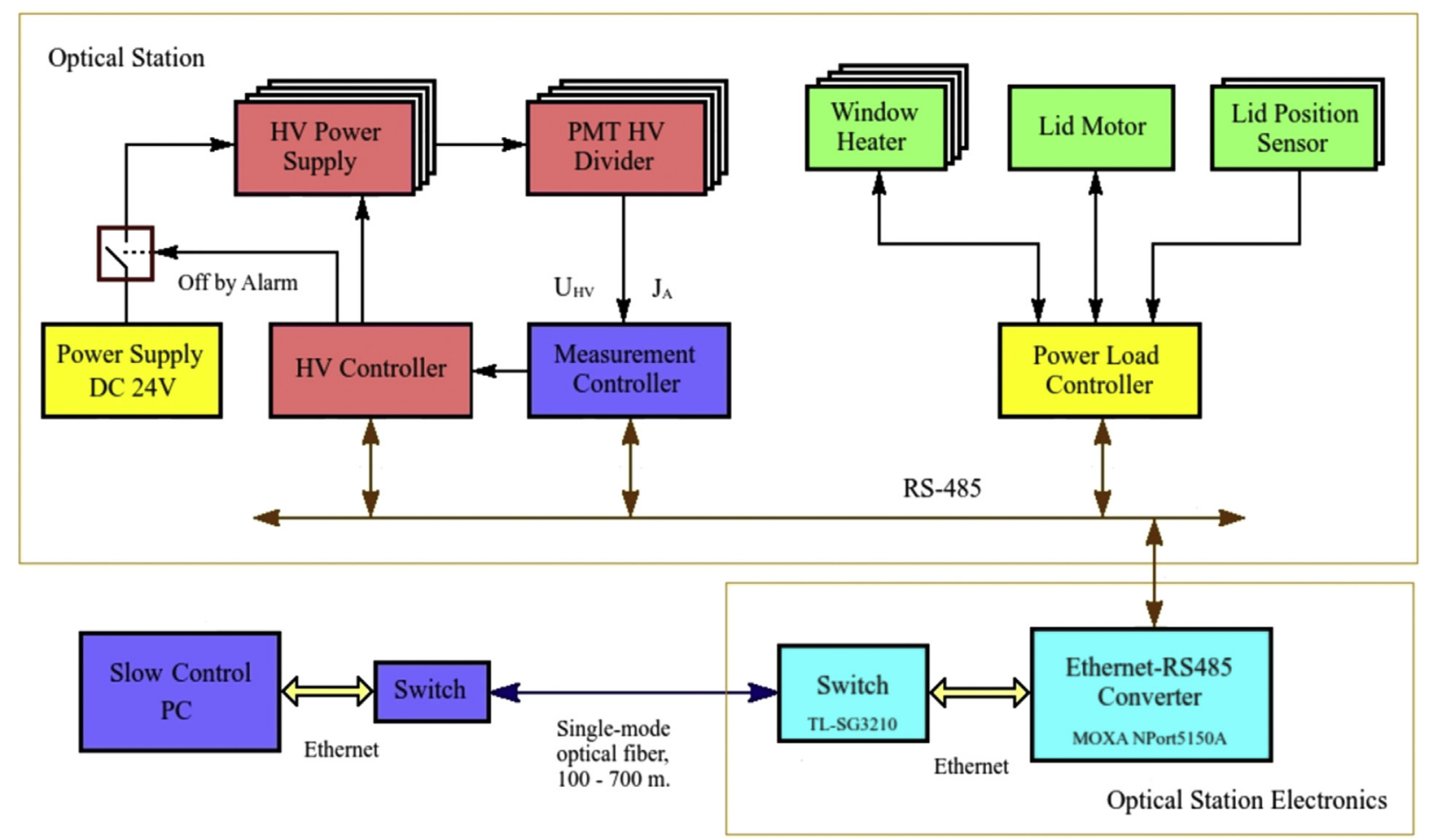

Fig. 4. Block diagram of the slow control system.

performs the initial start of the clock in the DRS- 4 boards when the data taking is started. The start time is recorded in the DRS- 4 board in an earlier time. MEGA-Host is the unit that keeps the GPS time and distributes $100 \mathrm{MHz}$ clock frequency to all Host-DRS via Super-Host.

Dead time of the DAQ is $\leq 0.5 \mathrm{~ms}$. The DAQ software was written in $\mathrm{C}++$ under OS Linux.

\section{Preliminary results of the EAS data processing}

To synchronize all stations of the array to sub-ns precision, a hybrid approach is used. It combines a custom-made synchronization technique (100 MHz clocks distributed over separate fibers from the array center; see above) and the White Rabbit (WR) Ethernet-based timing system [8]. A long-term direct cross-

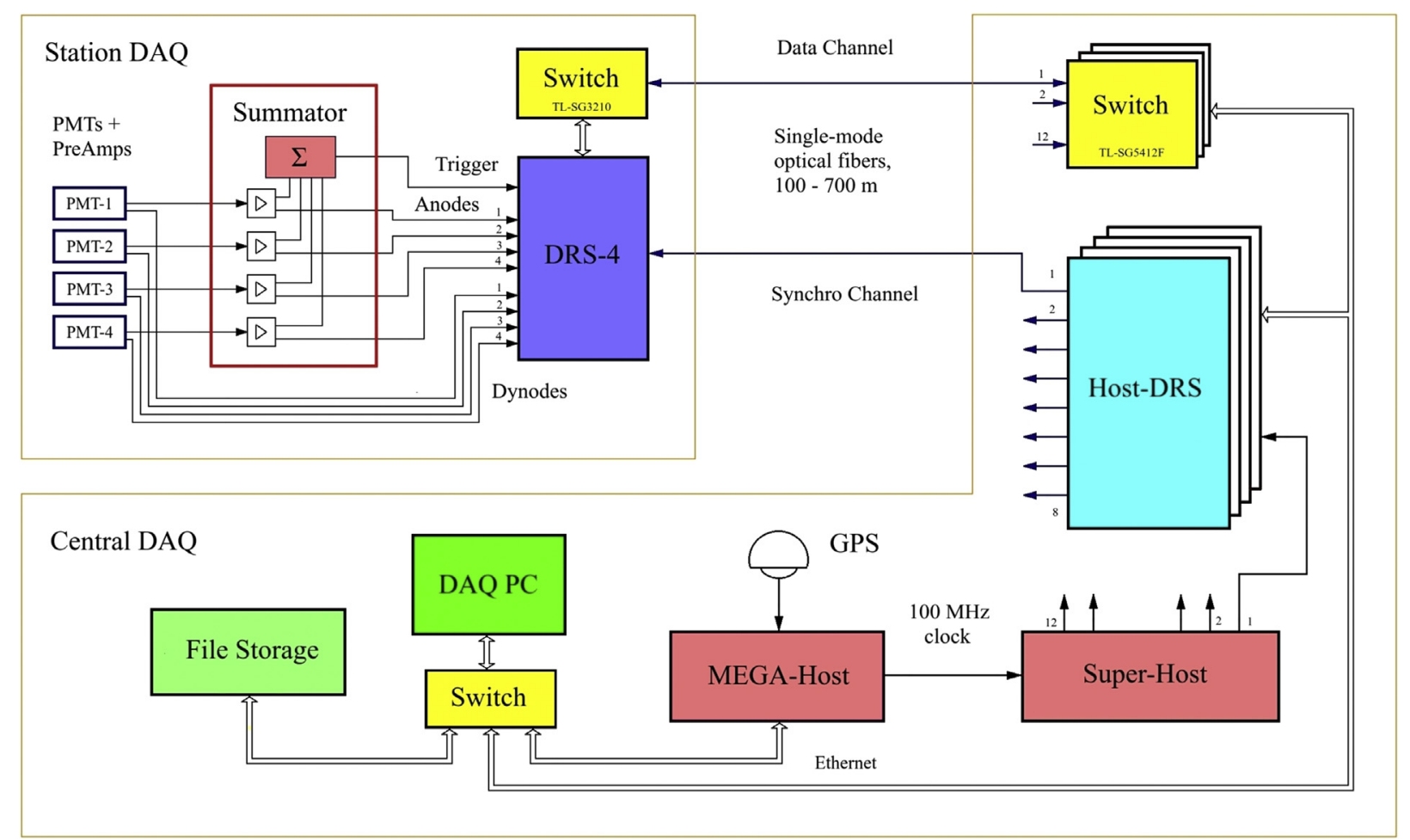

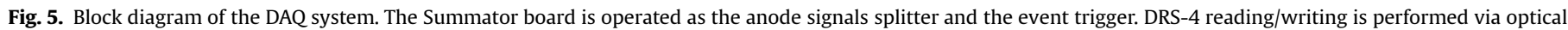
cable "Data Channel". The synchronization pulses are transmitted via optical cable "Synchro Channel". 
Timing stability: DAQBoard vs. WhiteRabbit

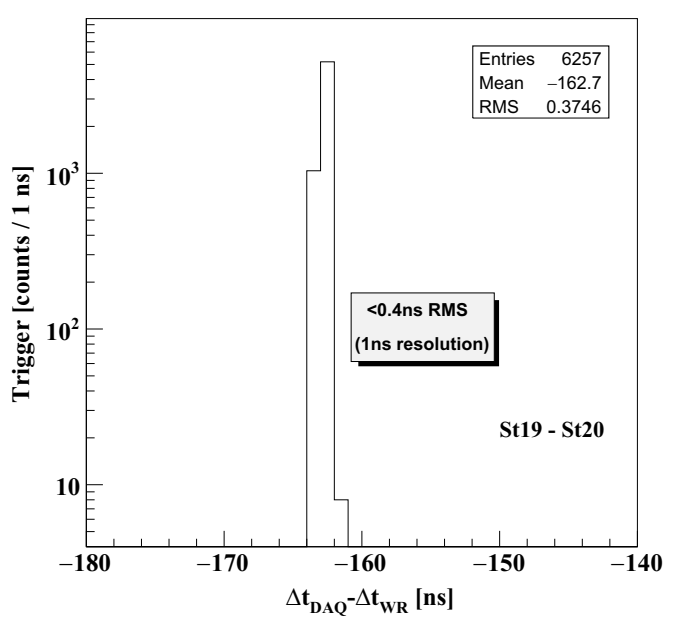

Shower front fit residuals

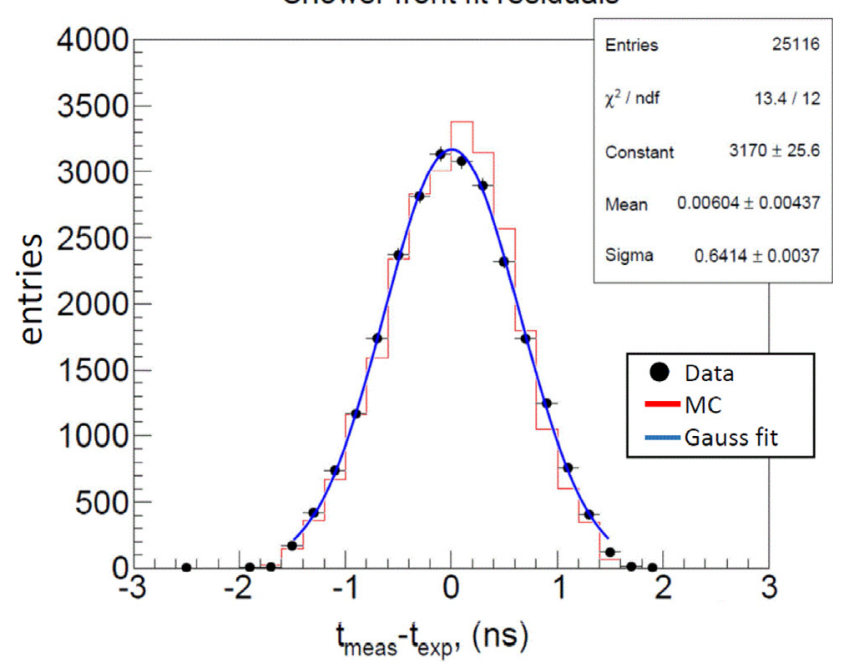

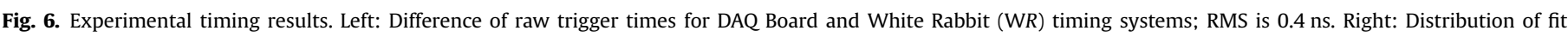
residuals for all HiSCORE stations after full EAS event reconstruction. Dots: data; histogram: simulated events; solid line: gaussian data fit [8].

verification of both clock systems was made. First results indicate very good precision with a long-term time relative synchronization of $\leq 0.4 \mathrm{~ns}$ rms, as shown for two stations in Fig. 6).

Methods for gamma-ray and cosmic-ray data reconstruction for HiSCORE are similar to those used for Tunka-133 [9,10]. The detector stations measure the light amplitudes and arrival time differences over a distance of few hundred meters. Signal amplitude, time differences, rise time and width of the arrival time distribution are used for the reconstruction of shower direction, axis position, primary energy, shower maximum height $X_{\max }$ and nature of the primary particle.

The integral amplitude spectrum of Cherenkov light flashes and Cherenkov light Lateral Distribution Function (LDF) are shown Fig. 7. Data are obtained with the 28-station TAIGA-HiSCORE array. The amplitude spectrum shows the integral air shower counting rate for an optical station as function of the measured amplitude. After EAS reconstruction, an LDF is given Fig. 7 - for an air shower found to have a primary energy of $3.3 \mathrm{PeV}$.

The expected accuracy of the air shower measurements near energy $100 \mathrm{TeV}$ is for the core location $\sim 7 \mathrm{~m}$, for the arrival direction $\sim 0.13^{\circ}$, for primary energy $\sim 14 \%$ and for $X_{\max } \sim 25 \mathrm{~g} / \mathrm{cm}^{2}$
[11].

\section{History of commissioning}

The history of the HiSCORE construction started in April 2012, when the first prototype station (2 PMTs) was deployed in the Tunka valley. Three detector stations were operating since October 2012. From 2013 to 2014, Tunka-HiSCORE (old name) was operated as a 9-station prototype array. The HiSCORE-9 array was arranged on a regular grid of $3 \times 3$ stations, with a side length of $300 \mathrm{~m}$. In Autumn 2014, the upgrade to 28 stations was started within the framework of the TAIGA collaboration. Since 2015, the 28-station array is in stable operation.

\section{Conclusion}

Construction and commissioning of the 28-station TAIGA-HiSCORE array was completed in September 2015. To increase the
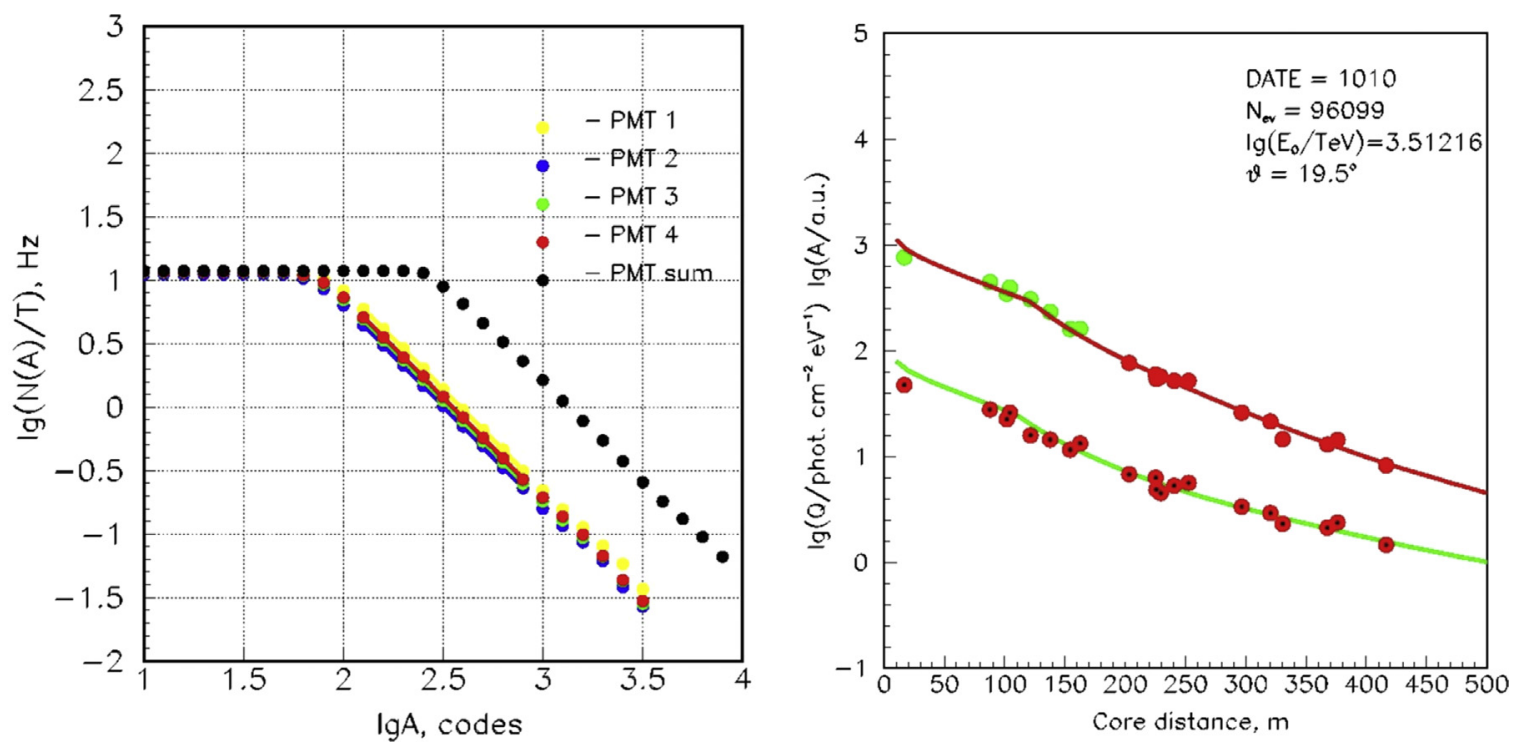

Fig. 7. Examples of the amplitude spectra (left) and Cherenkov light LDF (right), obtained after processing experimental EAS data. 
sensitivity it is planned to install more optical stations and instrument a larger area in the future.

The detection of sources of hard gamma rays with a spectrum up to several hundreds $\mathrm{TeV}$ would be direct evidence of acceleration of cosmic rays in these sources up to the knee in the all particle cosmic ray spectrum. Such Galactic PeV accelerators, or Pevatrons, have not been detected up to now [12].

\section{Acknowledgments}

This work was supported by the Russian Federation Ministry of Education and Science of the Russian Federation (Minobrnauka) (agreement 14.B25.31.0010, N 2014/51, project 1366, zadanie 3.889.2014/K), and the Grant 15-12-20022 of Russian Science Foundation (Sections 4 and 5).

\section{References}

[1] M. Tluczykont, et al., Gamma-ray and Cosmic Ray Astrophysics from $10 \mathrm{TeV}$ to $1 \mathrm{EeV}$ with the Large-Area $\left(>10 \mathrm{~km}^{2}\right)$ Air-Shower Detector SCORE, arXiv:astroph.IM/0909.0445v1.

[2] N.M. Budnev, et al., TAIGA the Tunka advanced instrument for cosmic ray physics and gamma astronomy - present status and perspectives, JINST 9 (2014) C09021, http://dx.doi.org/10.1088/1748-0221/9/09/C09021.
[3] I. Yashin, The Taiga project, J. Phys. Conf. Ser. 675 (3) (2016) 032037, http://dx doi.org/10.1088/1742-6596/675/3/032037.

[4] M. Tluczykont, D. Hampf, D. Horns, T. Kneiske, R. Eichler, R. Nachtigall, G. Rowell, The ground-based large-area wide-angle gamma-ray and cosmicray experiment HiSCORE, Adv. Space Res. 48 (2011) 1935-1941, http://dx.doi. org/10.1016/j.asr.2011.08.004 arXiv:1108.5880.

[5] S. Berezhnev, et al., The Tunka - multi-component EAS detector for high energy cosmic ray studies, Nucl. Instrum. Methods A732 (2013) 281-285, http: //dx.doi.org/10.1016/j.nima.2013.05.180.

[6] 〈http://www.digi.com/products/xbee-rf-solutions $\rangle$.

[7] 〈https://www.psi.ch/drs/〉.

[8] A. Porelli, et al., Timing calibration and directional reconstruction for TunkaHiSCORE, J. Phys. Conf. Ser. 632 (1) (2015) 012041 http://dx.doi.org/10.1088/ 1742-6596/632/1/012041;

R. Wischnewski, et.al., Proceedings of the 34th ICRC 2015, Proceedings of Science, PoS (ICRC2015) 1041.

[9] S. Berezhnev, et al., The Tunka-133 EAS Cherenkov light array: status of 2011 Nucl. Instrum. Methods A 692 (2012) 98-105, http://dx.doi.org/10.1016/j. nima.2011.12.091.

[10] V. Prosin, et al., Tunka-133: results of 3 year operation, Nucl. Instrum. Methods A 756 (2014) 94-101, http://dx.doi.org/10.1016/j.nima.2013.09.018.

[11] S. Berezhnev, et al., First results from the operation of the prototype TunkaHiSCORE array, Bull. Russ. Acad. Sci. Phys. 79 (3) (2015) 348-351;

S. Berezhnev, et al., Izv. Ross. Akad. Nauk Ser. Fiz. 79 (3) (2015) 381-384, http: //dx.doi.org/10.3103/S1062873815030107.

[12] J. Knödlseder, The future of Gamma-ray Astronomy. arXiv:astro-ph.IM/1602. 02728v1. 